\title{
The effect of learning on membership and welfare in an International Environmental Agreement
}

\author{
Larry Karp
}

Received: 17 March 2010 / Accepted: 20 April 2011 / Published online: 8 July 2011

(C) Springer Science+Business Media B.V. 2011

\begin{abstract}
Better information about the cost-benefit of abatement has an ambiguous effect on both the equilibrium membership and on aggregate welfare of an international environmental agreement. Previous papers claim that (complete) learning increases membership and decreases aggregate welfare. That claim is based on analysis of approximations to the relations between a damage parameter and membership and welfare. Those approximations have characteristics not shared by the functions they are intended to approximate, so conclusions based on the approximations are wrong. The correct result is that complete learning increases membership and welfare when the damage parameter is "very likely to be high", and the reverse holds when the damage parameter is "very likely to be low".
\end{abstract}

\section{Introduction}

A recent paper incorrectly claims that better information increases the equilibrium membership and reduces aggregate welfare in an International Environmental Agreement (IEA): Kolstad and Ulph (2008), which builds on Ulph (2004), Kolstad (2007) and Kolstad and Ulph (2008, unpublished working paper). This note explains and corrects the error, and it is of more general interest because it shows how to analyze the effect of better information on the formation of IEAs; it thereby contributes to two literatures, concerning the theory of international environmental agreements and concerning the effect of learning in games.

Electronic supplementary material The online version of this article (doi: 10.1007/s10584-011-0134-5) contains supplementary material, which is available to authorized users.

L. Karp $(\varangle)$

Department of Agricultural and Resource Economics, University of California,

Berkeley, CA, USA

e-mail:karp@berkeley.edu 
The papers cited above use a two-stage game consisting of a participation stage, when nations decide whether to join the IEA, and an abatement stage, when all nations decide whether to reduce emissions. They compare the equilibrium outcome when a damage parameter is uncertain at both stages (no learning), and the outcome when nature reveals the value of this parameter before either the participation or abatement decisions (complete learning). ${ }^{1}$

Ulph and Kolstad show that approximations of the functions relating the damage parameter to the equilibrium number of IEA members and to welfare are, respectively, convex and concave. They then apply Jensen's Inequality to establish their claim. Although the approximations have the curvature that the authors assert, the actual relations are neither convex nor concave. Therefore, Jensen's inequality is inapplicable: better information might increase equilibrium IEA size and decrease welfare, as claimed, or have the opposite effect.

The actual effect of learning on membership and welfare depends on the distribution of the damage parameter. I show that if the probability of low damages is small, then learning increases both expected membership and expected welfare; learning decreases both membership and welfare if the probability of low damages is large. For intermediate probabilities of low damages, learning can have opposite effects on membership and welfare.

\section{Preliminaries: known damage parameter}

In the standard model there are $N$ countries, each of which has a binary decision, to emit one unit of pollution or to abate. Emission creates one unit of private benefit for the country; for example, a country may have to sacrifice economic output in order to reduce emission. Each country suffers $\gamma$ units of damage for each unit of pollution emitted by any country, because the pollutant is global. The assumption $\gamma<1$ means that it is a dominant strategy for a country acting alone to emit. The assumption $\gamma N>1$ means that all countries are better off if all abate, compared to when all emit. The IEA instructs all members to abate only if there are enough members for the combined benefit of abatement to exceed the combined private costs.

If $m$ countries abate and $N-m$ countries emit, the payoffs of an abater and an emitter are, respectively, $-\gamma(N-m)$, and $1-\gamma(N-m)$. A nation that abates looses the 1 unit of private benefit associated with its pollution. If $m$ of the $N$ countries join an agreement and all members abate, each member obtains the payoff $-\gamma(N-m)$; if all members pollute, each member obtains the payoff $1-\gamma N$. Therefore, the IEA that maximizes the joint welfare of its members (as distinct from global, or aggregate welfare) instructs members to abate if and only if

$$
-\gamma(N-m) \geq 1-\gamma N,
$$

i.e. if and only if $m \geq \frac{1}{\gamma}$.

\footnotetext{
${ }^{1}$ The papers also consider a third information structure, "partial learning", in which nature reveals the damage parameter after the participation stage and before the abatement stage. The papers' result comparing partial learning to complete learning and to no learning do not rely on an approximation and they do not contain the error discussed here.
} 


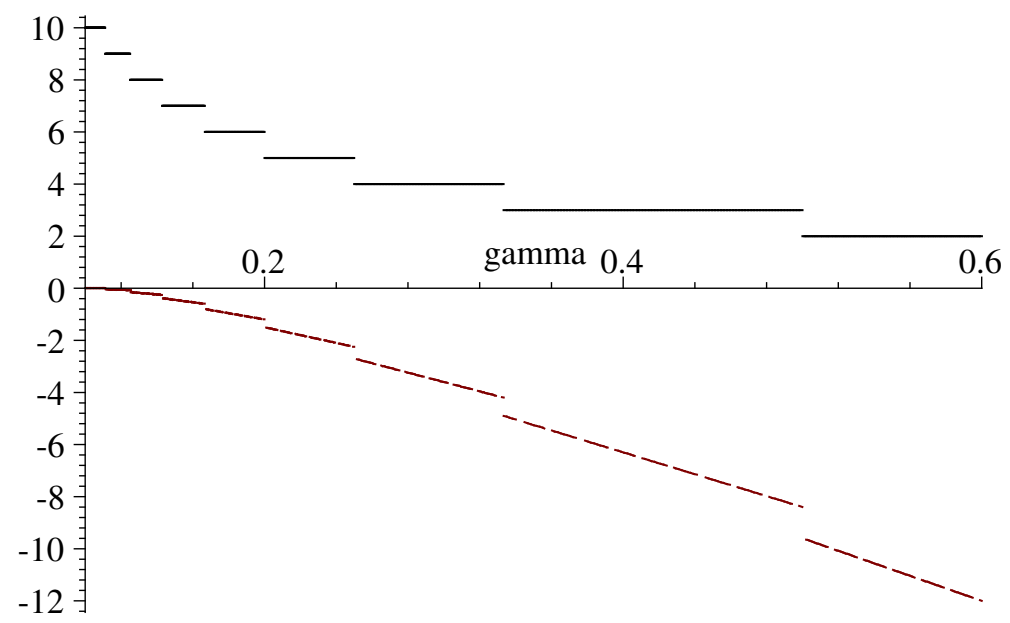

Fig. 1 Equlibrium membership (the solid step function in the top part of the figure) and welfare (the dashed graph in the lower part of the figure) as functions of $\gamma$

There are trivial Nash equilibria to the participation game, those with zero members or with a positive number of members who then decide not to abate. At the unique nontrivial Nash equilibrium to the participation game there are two or more IEA members and these decide to abate. This equilibrium consists of

$$
m^{*}=m(\gamma) \equiv h\left(\frac{1}{\gamma}\right)
$$

members, where $h(x)$, the ceiling function, equals the smallest integer not less than $x .^{2}$ When there are $m$ abaters, the combined benefit of polluters and abaters is $N-m-\gamma N(N-m)=(1-\gamma N)(N-m)$. Substituting $m^{*}$ in this expression gives global equilibrium welfare:

$$
W(\gamma)=(1-\gamma N)\left(N-h\left(\frac{1}{\gamma}\right)\right)
$$

Figure 1 graphs membership $m(\gamma)$, the step function in the top part of the graph, and scaled welfare $\frac{3}{N} W(\gamma)$, the negatively sloped discontinuous curve in the bottom

\footnotetext{
${ }^{2}$ If $m>m^{*}$ a signatory obtains a higher payoff by leaving the IEA, so $m>m^{*}$ is not a Nash equilibrium. If $m=m^{*}$ no signatory wants to defect, because doing so causes the IEA to decide not to abate, leaving the defector worse off. Moreover, no nonsignatory wants to join the IEA with $m=m^{*}$ members. Therefore $m=m^{*}$ is a Nash equilibrium. Any level of $m<m^{*}$ is a trivial equilibrium, because at this level, no abatement occurs in the next stage.
} 


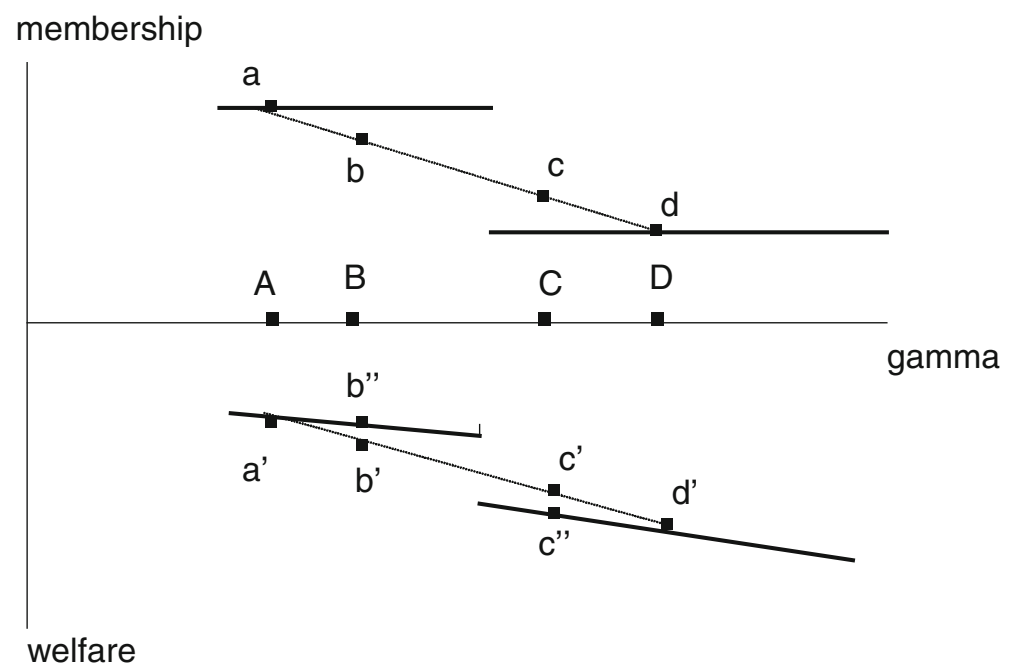

Fig. 2 Enlargement of Fig. 1: illustration of non-concavity and non-convexity

part of the curve, for $\gamma \in[0.1,0.6]$ and $N=10 .{ }^{3}$ The functions $m(\gamma)$ and $W(\gamma)$ are neither convex nor concave, although the former might appear "approximately convex" and the latter might appear "approximately concave". To demonstrate this non-convexity and non-concavity, Fig. 2 provides a close-up of Fig. 1 and adds to each graph a dotted line segment (between points $a$ and $d$ in the top part of the figure and between points $a^{\prime}$ and $d^{\prime}$ in the bottom part of the figure). These two line segments lie between, rather than always above or always below the discontinuous curves. Therefore, the functions corresponding to those curves are neither convex nor concave, and Jensen's inequality does not apply.

\section{Uncertainty about $\gamma$ and the effect of learning}

To introduce uncertainty, I use the assumption common to many papers, that the damage parameter has a two-point distribution: $\gamma$ equals $\gamma_{L}$ with probability $p$ and $\gamma_{H}$ with probability $1-p$, with $\gamma_{L}<\gamma_{H}$ If countries learn the true value before they make their participation decision ("complete learning"), the ex ante (before learning) expected equilibrium membership and expected aggregate welfare are

$$
\begin{aligned}
& m^{\text {learn }}=p h\left(\frac{1}{\gamma_{L}}\right)+(1-p) h\left(\frac{1}{\gamma_{H}}\right) \\
& W^{\text {learn }}=p W\left(\gamma_{L}\right)+(1-p) W\left(\gamma_{H}\right) .
\end{aligned}
$$

\footnotetext{
${ }^{3}$ Over this range, the assumptions $\gamma<1$ and $\gamma N \geq 1$ are satisfied. I use the scaling factor $\frac{3}{N}$ in the graph of $W$ so that the two graphs are on approximately the same scale. In the text I drop further reference to this scaling factor, since it plays no role except to improve the visuals.
} 
If they make their participation and abatement decisions before learning the value of $\gamma$, the equilibrium membership and expected welfare are ${ }^{4}$

$$
\begin{aligned}
m^{\text {no learn }} & =h\left(\frac{1}{p \gamma_{L}+(1-p) \gamma_{H}}\right) \\
W^{\text {no learn }} & =W\left(p \gamma_{L}+(1-p) \gamma_{H}\right) .
\end{aligned}
$$

The reduction in the expected number of members due to learning, denoted $\delta$, and the reduction in aggregate expected welfare due to learning, denoted $\Delta$, are

$$
\begin{aligned}
\delta & =m^{\text {no learn }}-m^{\text {learn }} \\
\Delta & =W^{\text {no learn }}-W^{\text {learn }} .
\end{aligned}
$$

In order to reduce the number of special cases, I adopt:

Assumption 1 Neither $\frac{1}{\gamma_{L}}$ nor $\frac{1}{\gamma_{H}}$ is an integer.

This assumption excludes a set of measure 0 from parameter space, so the results described in the following propositions are generic. The papers cited above claim that $\Delta>0>\delta$, i.e., learning increases membership and reduces welfare. Proposition 1 shows that the claim $0>\delta$ is true for small $p$ but false for large $p$. Proposition 2 shows that the claim $\Delta>0$ is false for small $p$ but true for large $p$. For completeness, parts (i) of both propositions consider the case where learning has no effect on the outcome. Parts (ii) of the propositions contain the significant results.

\section{Proposition 1 Adopt Assumption 1.}

(i) If $h\left(\frac{1}{\gamma_{H}}\right)=h\left(\frac{1}{\gamma_{L}}\right)$ then learning has no effect on equilibrium expected membership.

(ii) If $h\left(\frac{1}{\gamma_{H}}\right)<h\left(\frac{1}{\gamma_{L}}\right)$ then learning increases equilibrium expected membership $(\delta(p)<0)$ for small positive $p$ and decreases equilibrium expected membership $(\delta(p)>0)$ for large $p$.

\footnotetext{
${ }^{4}$ The ability to pass the expectations operator through the functions $h$ and $W$ depends on the fact that the model is linear in $\gamma$. At the abatement stage (given that $\gamma$ is uncertain) the IEA's decision depends on the number of members, which was determined at the earlier stage, and on the expectation of $\gamma, p \gamma_{L}+(1-p) \gamma_{H}$.

At the participation stage, each nation takes other nations' participation decisions as given, it takes as given the IEA's decision rule used in the next stage, and it maximizes its own expected payoff. Because of the linearity of the model, its decision depends on the expectation of $\gamma$. As a consequence, the equilibrium number of members, $m^{\text {no learn }}$, also depends only on this expectation.

Aggregate welfare depends directly on $\gamma$, but since welfare is linear in $\gamma$, expected welfare depends only on the expectation of $\gamma$. Aggregate welfare also depends on the number of members and on the IEA's decision rule, but these depend only on the expectation of $\gamma$, not on its realization.
} 
Proposition 2 Adopt Assumption 1.

(i) If $h\left(\frac{1}{\gamma_{H}}\right)=h\left(\frac{1}{\gamma_{L}}\right)$ then learning has no effect on equilibrium expected welfare.

(ii) If $h\left(\frac{1}{\gamma_{H}}\right)<h\left(\frac{1}{\gamma_{L}}\right)$ then learning increases equilibrium expected welfare $(\Delta(p)<0)$ for small positive $p$ and decreases equilibrium expected welfare $(\Delta(p)>0)$ for large $p$.

In the interest of completeness, the Appendix discusses in more detail the relation between my results and those of earlier papers. The Appendix also contains proofs of Propositions 1 and 2, but the intuition is readily apparent from Fig. 2. Let $\gamma_{L}=A$ and $\gamma_{H}=D$. These two values lie on either side of a point of discontinuity, and thus satisfy the necessary and sufficient condition for learning to have an effect on the equilibrium expected values of membership and welfare. For small $p$, the expectation of $\gamma$ is to the right of the point of discontinuity, close to point $D$, e.g. at point $C$. In the absence of learning, equilibrium membership equals the vertical coordinate of point $d$ and equilibrium welfare equals the vertical coordinate of point $c$ ". With learning, equilibrium expected membership equals the vertical coordinate of point $c$ and equilibrium expected welfare equals the vertical coordinate of point $c^{\prime}$. In this case, where $p$ is small, learning increases both expected membership and expected welfare. In contrast, if $p$ is large, then the expected value of $\gamma$ lies to the left of the point of discontinuity, e.g. at point $B$. In this case, the same kind of reasoning as above shows that learning decreases both expected membership and welfare.

\section{Conclusion}

Learning has an ambiguous effect on membership and welfare in an IEA game. The equilibrium must satisfy the integer constraint, the requirement that the number of countries that join the IEA be an integer. This constraint is central to the model, not an incidental feature of it. If nations were arbitrarily small (i.e. of measure 0) relative to the size of the world, then an individual nation's participation decision would have no effect on the equilibrium abatement decisions of IEA signatories. In that case, there would be no integer constraint. However, in that case, no nation would be "pivotal" and the only Nash equilibria that remain are trivial, i.e. they contain too few (possibly 0) members to induce abatement in the second stage of the game. In other words, the fact that nations have positive measure is responsible for the outcome that an equilibrium IEA has positive members. It is also responsible for the discontinuity of the graphs of equilibrium membership and welfare, as a function of a model parameter such as $\gamma$. This discontinuity (together with the slopes of the pieces) means that the graphs are neither concave nor convex. Therefore, the effect of learning has an ambiguous effect on both the equilibrium number of members and on equilibrium welfare.

Previous papers reached the wrong conclusion regarding the relation between (complete) learning and the equilibrium IEA size and aggregate welfare, because they relied on approximations that have properties not shared by the functions that are supposedly being approximated. The correct result, under the assumption of a two point distribution on the damage parameter, is that complete learning (when it has any effect) increases membership and welfare if the high damage outcome is 
"very likely"; learning decreases membership and welfare if the low damage outcome is "very likely". For intermediate probabilities, an example in the Appendix shows that learning might either reduce or increase expected membership and welfare. In addition, learning can have different effects on expected membership and welfare, possibly increasing one and decreasing the other.

Acknowledgement I thank Alistair Ulph for comments on an earlier version of this paper. The usual disclaimer holds.

\section{References}

Kolstad CD (2007) Systematic uncertainty in self-enforcing international environmental agreements. J Environ Econ Manage 53:68-79

Kolstad CD, Ulph A (2008) Learning and international environmental agreements. Clim Change 89:125-141

Ulph A (2004) Stable international environmental agreements with a stock pollutant, uncertainty and learning. J Risk Uncertain 29:53-73 